\title{
Una aproximación regional al alfabetismo financiero en México
}

\section{A regional approach to financial literacy in Mexico}

\author{
Osvaldo García Mata*
}

\begin{abstract}
This paper aims to analyze the determinants of financial literacy in Mexico with a regional approach. Its originality lies in emphasizing the regional differences in financial attitude, financial behavior, and financial knowledge. It uses ordered logit models constructed with data from the National Survey on Financial Inclusion 2018. The results confirm that education is essential for financial literacy, but other factors have different effects in each region. Its main limitation is the lack of data to perform a longitudinal analysis to observe how contextual changes affect financial literacy. Its findings can help focalize the financial inclusion policy.
\end{abstract}

Keywords: financial literacy, financial inclusion, education, region.

\section{Resumen}

El objetivo de esta investigación es analizar los determinantes del alfabetismo financiero en México con un enfoque regional. Su originalidad reside en enfatizar las diferencias regionales en actitud, comportamiento y conocimiento financieros. Utiliza modelos logit ordenados construidos con datos de la Encuesta Nacional de Inclusión Financiera 2018. Se confirma que la educación es esencial para el alfabetismo financiero; sin embargo, otros factores tienen diferentes efectos en cada región. Entre sus limitaciones está la falta de datos para analizar longitudinalmente cómo los cambios en el entorno afectan al alfabetismo financiero. Sus hallazgos pueden aprovecharse para focalizar la política de inclusión financiera.

Palabras clave: alfabetismo financiero, inclusión financiera, educación, región.

\footnotetext{
* Universidad Autónoma de Tamaulipas, correo-e: ogarciam@uat.edu.mx
} 


\section{Introducción}

La Organización para la Cooperación y el Desarrollo Económicos (OCDE) define alfabetismo financiero como "una combinación de conocimientos, habilidades, actitudes y comportamientos necesarios para tomar decisiones financieras sólidas, con el fin último de alcanzar el bienestar financiero individual" (OCDE/INFE, 2011: 3). Éste facilita administrar el dinero con mayor eficiencia, tomar decisiones económicas confiables y planificar mejor el futuro financiero (Altman, 2012; Clark et al., 2006; Lusardi y Mitchell, 2011a). Bajo condiciones macroeconómicas estables, promueve el ahorro, la inversión, el emprendimiento y la acumulación de capital, y durante épocas de estrés económico, ayuda a las personas a sobrellevar la situación (Ćumurović y Hyll, 2019; Klapper et al., 2013).

El alfabetismo financiero es un elemento necesario mas no el único para mejorar el bienestar financiero. Estudiarlo desde un enfoque regional es importante porque las características culturales, económicas, sociales y de infraestructura del entorno pueden vincularse al desempeño financiero de la población (Boisclair et al., 2017; Cucinelli et al., 2019). Propiciar que la gente aplique con éxito sus conocimientos financieros requiere diseñar e instrumentar iniciativas de educación más eficaces y eficientes que, entre otros aspectos, consideren estas diferencias regionales (Bernheim et al., 2001; Carpena et al., 2017; Cude, 2010).

El alfabetismo financiero en México ha sido poco investigado. Como parte de un estudio internacional llevado a cabo en más de 140 naciones, Klapper et al. (2015) estimaron para este país una tasa de alfabetismo financiero de 32\% entre personas de 15 años y más. Villagómez y González (2014) analizaron si éste puede influir en un incremento del ahorro para el retiro. Villagómez (2016) y Arceo-Gomez y Villagómez (2017) midieron su nivel entre estudiantes de preparatoria del área metropolitana de la Ciudad de México y determinaron que éste es deficiente. Villagómez e Hidalgo (2017) investigaron su relación con las habilidades matemáticas entre jóvenes de 15 a 18 años en la misma zona y concluyeron que éstas guardan una relación estrecha con el alfabetismo financiero. La mayoría de estos estudios se enfocaron en un área urbana donde habita no más del $21 \%$ de la población total de México y que por sus características sociodemográficas es distinta a otras regiones del país (Inegi, 2015). Recientemente, Antonio-Anderson et al. (2020) analizaron el alfabetismo financiero con un enfoque nacional, aunque sin distinguir entre sus componentes.

Adicionalmente, la Política Nacional de Inclusión Financiera (CNBV, 2020) consigna las amplias brechas en materia de competencias financieras entre las distintas entidades y regiones del país, y la necesidad de 
focalizar los esfuerzos para educar financieramente a la población de acuerdo con sus características específicas.

En este trabajo se explora el impacto de variables sociodemográficas específicas sobre los tres componentes del alfabetismo financiero en México, con énfasis en las diferencias regionales y locales. Se propone responder a las siguientes preguntas: ¿̇on los mismos factores socioeconómicos los que determinan el nivel de alfabetismo financiero para toda la población del país o hay determinantes de mayor peso en algunas regiones? De haber diferencias, ¿qué factores deberían considerarse para adecuar las iniciativas que se desprendan de la Política Nacional de Inclusión Financiera a las diferentes regiones de México?

Identificar estos determinantes puede ser de utilidad en el ámbito público para instrumentar estrategias efectivas derivadas de las políticas que estimulan la inclusión financiera y, por ende, el desarrollo económico y social. En el ámbito empresarial puede aprovecharse para diseñar productos financieros especializados en atender demandas locales.

Los componentes a los cuales se refiere esta investigación son conocimiento financiero $(F K I)$, actitud financiera $(F A I)$ y comportamiento financiero $(F B I)$. Se emplea un método de análisis factorial y modelos logit ordenados con los que se estiman índices para cada componente a partir de algunas variables socioeconómicas: género, edad, nivel educativo, estado civil, tamaño de la localidad y nivel de ingreso.

El enfoque de la investigación es cuantitativo. Toma como base los datos de la Encuesta Nacional de Inclusión Financiera, ENIF (Inegi, 2019a), correspondientes a 2018. El estudio es de tipo descriptivocorrelacional. Al probar si los factores determinantes del alfabetismo financiero en México son distintos para cada región, se justifica la instrumentación de estrategias y programas adecuados a los contextos regionales y locales en México.

Este documento está dividido en cuatro secciones. Primero se hace un repaso de los estudios sobre alfabetismo financiero que se han efectuado en México. En la siguiente sección se describen los datos y modelos empleados en el análisis. Posteriormente se exponen y comentan los resultados obtenidos. Para finalizar, se presentan las conclusiones y se sugieren futuras líneas de investigación.

\section{Medición del alfabetismo financiero}

Para medir el alfabetismo financiero, la Organización para la Cooperación y el Desarrollo Económicos recomienda evaluar a las personas en tres dimensiones: conocimiento, actitud y comportamiento financieros 
(OCDE/INFE, 2011). El conocimiento financiero se refiere al dominio que un individuo tiene de conceptos financieros básicos y su habilidad para utilizarlos en situaciones de decisión (Atkinson y Messy, 2012). En este sentido se observa una diferencia entre la comprensión y la aplicación del conocimiento, en donde la segunda tiene sentido sólo cuando las personas son capaces de usar sus conocimientos con confianza y efectividad (Bay et al., 2014; Huston, 2010; Remund, 2010).

La actitud financiera está relacionada con la predisposición a decidir y actuar en materia financiera; una actitud positiva considera el futuro en la priorización de metas, la previsión ante emergencias y la planeación para el retiro (Atkinson y Messy, 2012). El comportamiento financiero da cuenta de prácticas tendientes a mejorar el bienestar financiero, tales como la planeación de los gastos personales y familiares, el establecimiento y consecución de metas, el uso responsable del crédito y la sujeción a un presupuesto (Atkinson y Messy, 2012).

Aunque fuera de México, Huston (2010) llevó a cabo una amplia revisión sobre modelos para medir el alfabetismo financiero. Analizó 71 trabajos publicados entre 1996 y 2008, principalmente en Estados Unidos, por 50 diferentes autores y organizaciones. Una revisión no tan amplia pero más reciente es la efectuada por Das (2016), quien revisó 14 trabajos publicados entre 2002 y 2015. En ambas encontraron que la mayoría de los estudios se especializan en la evaluación del conocimiento financiero y pocos incluyen actitud y comportamiento.

Durante 2014, Klapper et al. (2015) recolectaron datos de más de 150 mil personas de 15 años o más, en 140 países, para medir el alfabetismo financiero global. Consideraron cuatro aspectos básicos del conocimiento financiero: inflación, diversificación de riesgo, interés simple e interés compuesto. Decidieron llamar alfabetas financieros a aquellos que obtuvieron tres o más aciertos de los cuatro aspectos evaluados. Encontraron que el alfabetismo financiero en el mundo es de $33 \%$, mientras que en México es de 32\%. Este resultado lo colocaría en el rango de las economías emergentes, representadas por Brasil, Rusia, India, China y Sudáfrica (BRICS), en donde los valores nacionales fluctúan desde $24 \%$ hasta $42 \%$. En contraste, las economías del G-7, integrado por Estados Unidos de América, Canadá, Gran Bretańa, Francia, Alemania, Italia y Japón, alcanzan tasas que varían entre 37 y 68 por ciento.

Villagómez y González (2014) analizaron si el monto de riqueza de una persona en México puede incrementarse cuando ésta posee mayor nivel de alfabetismo financiero. Utilizaron el modelo propuesto por Atkinson y Messy (2012) y los datos obtenidos de la Encuesta Nacional sobre los Niveles de Vida de los Hogares 2005. Construyeron dos índices que consideraron los componentes de actitud y comportamiento, pero 
no el de conocimiento. No obtuvieron datos suficientes para incluir el conocimiento financiero. Tras analizar los datos, concluyeron que el alfabetismo financiero tiene un efecto significativo y positivo en la acumulación de la riqueza individual.

Villagómez (2016) midió el nivel de alfabetismo financiero a partir de una muestra de 889 estudiantes preparatorianos, entre 15 a 18 años, de la zona metropolitana del Valle de México. Utilizó las metodologías de la OCDE a través de la Red Internacional de Educación Financiera (OCDE/ INFE, 2011) y de Lusardi y Mitchell (2011b) para evaluar actitud, comportamiento y conocimiento financieros. Observó ligeras diferencias en los resultados por género, a favor de los hombres, y por tipo de escuela, a favor de la educación privada.

En otro estudio, Arceo y Villagómez (2017) estimaron el alfabetismo financiero de jóvenes estudiantes de preparatoria del área metropolitana de la Ciudad de México, mediante la evaluación de ocho preguntas sobre conocimiento, ocho sobre comportamiento y tres sobre actitud financiera. Encontraron que sólo 20\% comprende los conocimientos financieros básicos, $57 \%$ sigue un comportamiento financiero deseable y $70 \%$ tiene una actitud financiera positiva.

Villagómez e Hidalgo (2017) investigaron la relación entre el alfabetismo financiero y las habilidades matemáticas de jóvenes estudiantes del valle de México. Las variables independientes que utilizaron fueron la puntuación obtenida en la sección matemática del instrumento que aplicaron y los niveles de confianza autopercibidos en el manejo de las matemáticas y las finanzas. Confirmaron que las habilidades cuantitativas tienen una influencia significativa y positiva en los índices de conocimiento, actitud y comportamiento financiero. Tanto en éste como en los estudios realizados en México mencionados anteriormente sobresale el papel determinante de la educación financiera en el desarrollo económico y el bienestar de las personas.

A partir de los resultados de la ENIF 2018 (Inegi, 2019a), AntonioAnderson et al. (2020) analizaron los factores determinantes del alfabetismo financiero en México. En su estudio incluyeron la variable región, aunque utilizaron un índice general sin distinguir sus componentes. Otros estudios han demostrado que estos componentes pueden variar de manera inversa entre sí, de tal forma que uno podría contrarrestar el efecto de otro (Atkinson y Messy, 2012; Baglioni et al., 2018; Cucinelli et al., 2019).

En la presente investigación se propone medir el alfabetismo financiero y proporcionar evidencia que demuestre que $\left(\mathrm{H}_{1}\right)$ en México prevalece un nivel de conocimiento financiero bajo en todas sus regiones, en comparación con el observado por Klapper et al. (2015) en naciones desarrolladas, pero comparable al de economías emergentes; y que $\left(\mathrm{H}_{2}\right)$ los 
determinantes socioeconómicos del alfabetismo financiero varían de región a región, en cada uno de sus tres componentes: conocimiento, actitud y comportamiento (Cucinelli et al., 2019). Al comprobar lo anterior, esta investigación puede servir como evidencia para justificar que la instrumentación de la Política Nacional de Inclusión Financiera debe considerar algunos elementos generales y otros de carácter local en los que las entidades y municipios podrían tener mayor participación.

\section{Datos y metodología}

La Encuesta Nacional de Inclusión Financiera (ENIF) tiene el objetivo de medir el acceso y uso de los servicios financieros en México. Es llevada a cabo por la Comisión Nacional Bancaria y de Valores (CNBV) y el Instituto Nacional de Estadística y Geografía (Inegi). En su tercera edición, efectuada del 30 de abril al 22 de junio de 2018, incluyó entrevistas directas a habitantes de 14,500 viviendas, seleccionadas de manera probabilística y estratificada. Por primera ocasión consignó datos representativos a nivel regional y sobre capacidades financieras (Inegi, 2019a).

En 2018, la ENIF (Inegi, 2019a) estableció seis regiones geográficas con características contrastantes, que podrían presentar factores estructurales asociados al alfabetismo financiero, como se aprecia en el cuadro 1.

La Ciudad de México (R4), con 7.0\% de la población nacional total y $8.1 \%$ de la Población Económicamente Activa (PEA) contribuye con $16.5 \%$ del Producto Interno Bruto, PIB. Su porcentaje de población en pobreza extrema es el más bajo entre regiones; sin embargo, presenta una tasa de informalidad laboral media y de desocupación alta.

La contribución de las regiones Noroeste (R1) y Noreste (R2) al PIB es ligeramente superior a su participación en la población total y la PEA del país. Registran tasas de informalidad laboral y pobreza extrema inferiores a las de las regiones del sur.

La región Sur (R6) es la de mayor porcentaje de población en pobreza extrema y de informalidad laboral, pero la de menor tasa de desocupación. La región Occidente y Bajío (R3) presenta una tasa de desocupación baja y de informalidad laboral media, en tanto que la Oriente y Centro Sur (R5) es la segunda con mayor tasa de informalidad laboral pero con una desocupación comparable a la de las regiones norteñas.

Aunque estas características socioeconómicas también pueden presentar alta variabilidad hacia el interior de las regiones, no sólo los rasgos individuales sino las diferencias interregionales podrían influir en el desempeño financiero, tal como lo observaran Cucinelli et al. (2019) en Italia. 


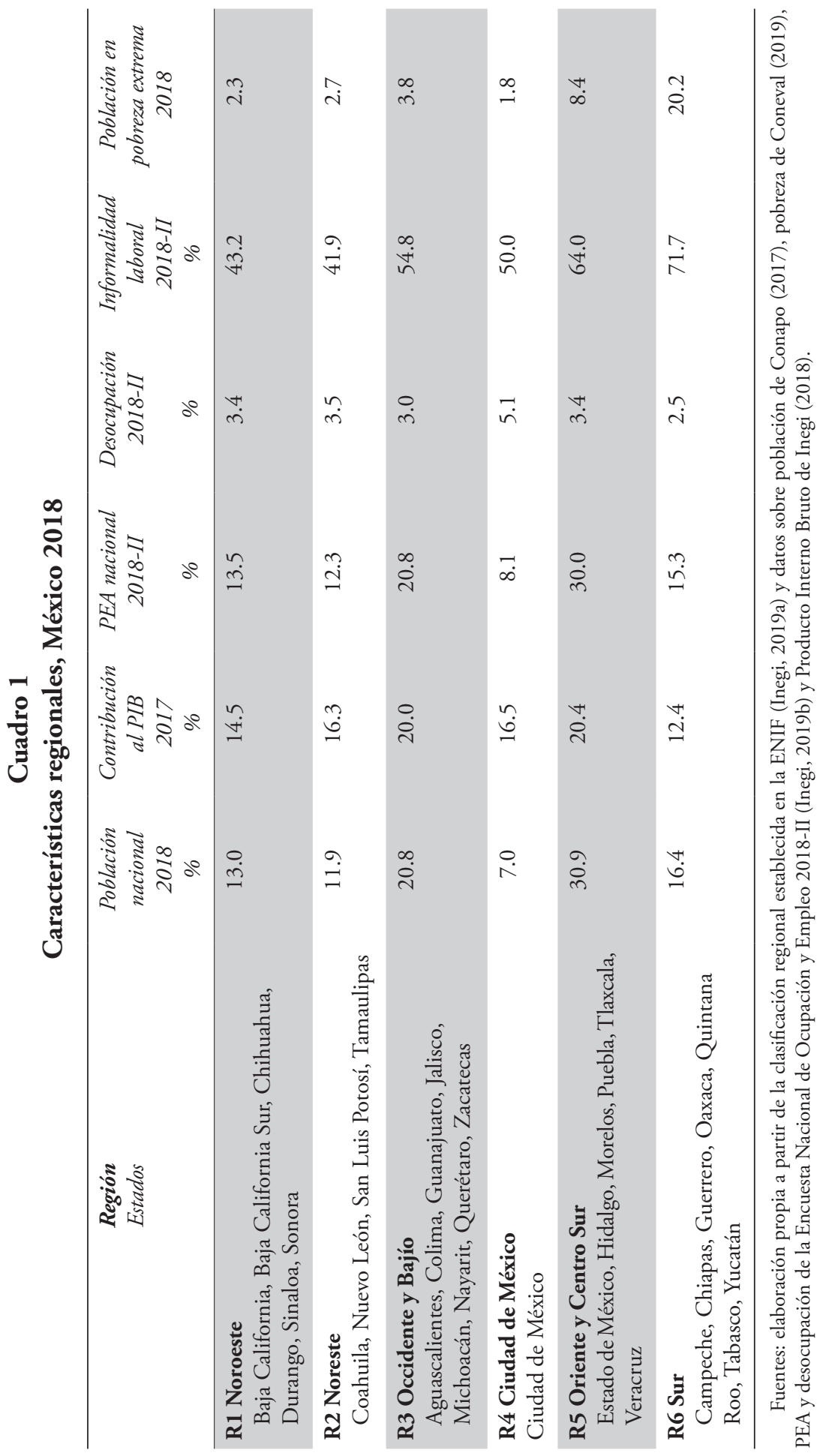


Para esta investigación se analizaron 12,446 registros, 54.7\% correspondientes a mujeres y $45.3 \%$ a hombres, entre 18 y 70 años de edad, $48.4 \%$ residentes en localidades de 100,000 habitantes o más. El número de observaciones por característica de la muestra se puede observar en el cuadro 2.

\section{Cuadro 2}

Características de la muestra, México 2018

\begin{tabular}{|c|c|c|}
\hline Caracteristicas & $N$ & $\%$ \\
\hline \multicolumn{3}{|l|}{ Género } \\
\hline Femenino & 6807 & 54.7 \\
\hline Masculino & 5639 & 45.3 \\
\hline \multicolumn{3}{|l|}{ Edad } \\
\hline $18-23$ & 1518 & 12.2 \\
\hline $24-29$ & 1680 & 13.5 \\
\hline $30-35$ & 1806 & 14.5 \\
\hline $36-41$ & 1786 & 14.3 \\
\hline $42-47$ & 1610 & 12.9 \\
\hline $48-53$ & 1364 & 11.0 \\
\hline $54-59$ & 1103 & 8.9 \\
\hline $60-65$ & 955 & 7.7 \\
\hline $66-70$ & 624 & 5.0 \\
\hline \multicolumn{3}{|l|}{ Máximo grado de estudios } \\
\hline Ninguna & 484 & 3.9 \\
\hline Preprimaria o primaria & 2845 & 22.9 \\
\hline Secundaria & 3925 & 31.5 \\
\hline Preparatoria & 2550 & 20.5 \\
\hline Licenciatura o equivalente & 2382 & 19.1 \\
\hline Posgrado & 253 & 2.0 \\
\hline No respondió & 7 & 0.1 \\
\hline
\end{tabular}

Ingreso anual en miles de pesos

\begin{tabular}{lrc}
\hline Sin ingresos & 3951 & 31.7 \\
Más de 0 hasta 40 & 1848 & 14.8 \\
Más de 40 hasta 60 & 1540 & 12.4 \\
Más de 60 hasta 80 & 1770 & 14.2 \\
Más de 80 hasta 100 & 750 & 6.0 \\
\hline
\end{tabular}


Cuadro 2 (continuación)

\begin{tabular}{|c|c|c|}
\hline Caracteristicas & $N$ & $\%$ \\
\hline Más de 100 hasta 120 & 428 & 3.4 \\
\hline Más de 120 hasta 140 & 439 & 3.5 \\
\hline Más de 140 hasta 160 & 301 & 2.4 \\
\hline Más de 160 & 698 & 5.6 \\
\hline No respondió & 721 & 5.8 \\
\hline \multicolumn{3}{|l|}{ Estado civil } \\
\hline Soltero (incluye separado, viudo, divorciado) & 4547 & 36.5 \\
\hline Casado (incluye unión libre) & 7899 & 63.5 \\
\hline \multicolumn{3}{|l|}{ Tamańo de la localidad } \\
\hline Menos de 2500 & 2825 & 22.7 \\
\hline $2500-14,999$ & 1751 & 14.1 \\
\hline $15,000-99,999$ & 1847 & 14.8 \\
\hline 100,000 o más & 6023 & 48.4 \\
\hline \multicolumn{3}{|l|}{ Región } \\
\hline Noroeste & 2304 & 18.5 \\
\hline Noreste & 2255 & 18.1 \\
\hline Occidente y Bajío & 2348 & 18.9 \\
\hline Ciudad de México & 877 & 7.0 \\
\hline Centro Sur y Oriente & 2345 & 18.8 \\
\hline Sur & 2317 & 18.6 \\
\hline Total & 12,446 & 100.0 \\
\hline
\end{tabular}

Fuente: elaboración propia con base en datos de la ENIF (Inegi, 2019a).

De la ENIF se aprovecharon 14 reactivos para estimar, con apego a lo establecido por la OCDE en la guía de la Red Internacional de Educación Financiera (OCDE/INFE, 2011), los tres índices componentes del alfabetismo financiero: conocimiento financiero $(F K I)$, actitud financiera $(F A I)$ y comportamiento financiero $(F B I)$.

Para el índice de conocimiento financiero $(F K I)$ se tomaron en cuenta siete reactivos clasificados, de acuerdo con el modelo utilizado por Klapper et al. (2015), en cuatro grupos: inflación ( $F K Q 1$ y $F K Q 2)$, diversificación del riesgo ( $F K Q 3$ y FKQ4), interés simple (FKQ5 y FKQ6) e interés compuesto (FKQ7). La ecuación (1) muestra el cálculo de este índice. 


$$
\begin{gathered}
F K I=F K Q 1 * F K Q 2+F K Q 3 * F K Q 4+F K Q 5 * F K Q 6+F K Q 7 ; \\
F K I \in[0,4]
\end{gathered}
$$

En este índice, los ítems $F K Q i$, para $i=1 \ldots 7$ son variables binarias que adoptan un valor de uno si la pregunta fue correctamente contestada y cero para respuestas incorrectas o cuando la persona admite no saber cuál es la respuesta correcta. En este sentido, sólo se consideran los registros sobre los que se tiene certeza de si el individuo conoce o no la respuesta correcta. El registro se omite si el encuestado no respondió.

La inflación, diversificación del riesgo e interés simple requieren que se responda correctamente a los dos reactivos para evaluar positivamente el factor en cuestión. Es decir, se asume que una persona entiende el concepto de inflación si y sólo si proporciona respuestas correctas a los ítems $F K Q 1$ y $F K Q 2$.

Siguiendo la definición acuñada por Klapper et al. (2015), una persona es alfabeta financiera si obtiene al menos tres aciertos de los cuatro factores evaluados. Para esta investigación se establece que si $F K I \geq 3$ entonces la persona se considera alfabeta desde una perspectiva de conocimiento financiero.

El índice de actitud financiera se calcula con la suma de los valores asociados a los ítems $F A Q 1$ y FAQ2, como se indica en la ecuación (2). Con ellos se evalúa si el encuestado piensa en el futuro antes de gastar su dinero, siguiendo el modelo de Cucinelli et al. (2019). El ítem FAQ1 se refiere al dilema entre ahorro y consumo presente. Toma un valor de dos si quien responde prefiere siempre ahorrar en lugar de gastar, uno si lo prefiere algunas veces y cero si prefiere gastar en lugar de ahorrar. El ítem $F A Q 2$ evalúa la predisposición a las compras por impulso. Toma un valor de dos cuando el encuestado siempre considera si puede pagar algo antes de comprarlo, uno si lo considera algunas veces y cero si nunca lo hace. Si la persona no sabe o no contesta, el registro no se toma en cuenta.

$$
F A I=F A Q 1+F A Q 2 ; F A I \in[0,4]
$$

De manera análoga al criterio establecido por Klapper et al. (2015) para el conocimiento financiero, se define a un alfabeta desde la perspectiva de actitud financiera si $F A I \geq 3$.

El índice de comportamiento financiero $(F B I)$ se calcula mediante la suma de los valores asociados a cinco ítems, como puede apreciarse en la ecuación (3). Dos se refieren a los hábitos de planeación financiera: si la persona sigue un presupuesto $(F B Q 1)$ y con qué frecuencia se propone metas financieras de largo plazo $(F B Q 2)$. Tres evalúan el cumplimiento de compromisos financieros: si lo que gana le alcanza para cubrir sus 
gastos $(F B Q 3)$, si podría solventar una emergencia económica con sus ahorros $(F B Q 4)$ y con qué frecuencia paga sus cuentas a tiempo ( $F B Q 5)$.

Los ítems $F B Q 1, F B Q 3$ y $F B Q 4$ toman el valor de uno en caso afirmativo y cero en caso negativo. Para los ítems $F B Q 2$ y $F B Q 5$, se asignó el valor de uno a las respuestas siempre y algunas veces, y de cero a nunca. El registro se omite si el encuestado no sabe o no responde.

$$
F B I=F B Q 1+F B Q 2+F B Q 3+F B Q 4+F B Q 5 ; F B I \in[0,5]
$$

Para definir a un alfabeta financiero desde la perspectiva del comportamiento, se estableció un criterio de al menos cuatro respuestas correctas de cinco posibles $(F B I \geq 4)$.

Aunque pareciera natural integrar los tres índices en uno solo, para esta investigación se optó por estudiarlos por separado debido a que evalúan diferentes dimensiones del individuo y a que, en apego al criterio utilizado por Cucinelli et al. (2019) y por Atkinson y Messy (2012), se asumió que su desempeño obedecería de manera distinta al mismo conjunto de variables socioeconómicas.

Para cada uno de estos índices se construyeron modelos econométricos en los que se prueba si es posible explicar su variabilidad a través de características sociodemográficas, de manera regional. Con éstos se buscó determinar si existe algún efecto significativo en cada una de las variables dependientes ( $F K I$, FAI y $F B I$ ) relacionado con las variables independientes: género, Gen, edad, $E d a d$, nivel educativo, $E d u c$, nivel de ingreso, Ingr, y estado civil, EstC (Almenberg y Widmark, 2011; Boisclair et al., 2017; Cucinelli et al., 2019; Klapper et al., 2015; Potrich et al., 2015; Van Rooij et al., 2011). Adicionalmente se incluyó como variable de control el tamaño de la localidad, TLoc (Boisclair et al., 2017; Cucinelli et al., 2019). Lo anterior se resume en el cuadro 3.

Para analizar las variables dependientes correspondientes a los índices de conocimiento, actitud y comportamiento financiero, por separado, se construyeron modelos logit ordenados. El método de mínimos cuadrados ordinarios no es recomendable cuando la variable dependiente es ordinal (Leckie et al., 2016), en este caso porque la diferencia entre el número de aciertos obtenidos a nivel individual, si bien refleja cierto grado de orden, sólo tiene significado relativo, y porque dos de los tres índices presentan una distribución sesgada.

Para cada índice se construyeron modelos regionales con la intención de observar y comparar sus factores determinantes, expresados por un conjunto de variables sociodemográficas. De esta manera, para la persona $i$ de la región $j=1 \ldots \sigma$, el modelo está definido por la ecuación (4): 


\section{Cuadro 3}

Descripción de variables

\begin{tabular}{|c|c|c|}
\hline Variable & Descripción & Fuente \\
\hline \multicolumn{3}{|c|}{ Variables dependientes } \\
\hline $\begin{array}{l}\text { Índice de } \\
\text { Conocimiento } \\
\text { Financiero } \\
(F K I)\end{array}$ & $\begin{array}{l}\text { Variable ordinal calculada a partir de } \\
\text { preguntas binarias, (uno si la respuesta es } \\
\text { correcta, cero si es incorrecta o no sabe), se } \\
\text { refiere a inflación (FKQ1 y FKQ2), diversifi- } \\
\text { cación del riesgo (FKQ3 y FKQ4), interés } \\
\text { simple }(F K Q 5 \text { y } F K Q 6) \text { e interés compuesto } \\
(F K Q 7) . F K I=F K Q 1 * F K Q 2+F K Q 3 * F K Q 4 \\
+F K Q 5 * F K Q 6+F K Q 7 ; F K I \in[0,4]\end{array}$ & $\begin{array}{l}\text { Propuesta del } \\
\text { autor con base en } \\
\text { Klapper } \text { et al. } \\
(2015) \text {. }\end{array}$ \\
\hline $\begin{array}{l}\text { Índice de } \\
\text { Actitud } \\
\text { Financiera } \\
(F A I)\end{array}$ & $\begin{array}{l}\text { Variable ordinal. Las actitudes esperadas } \\
\text { reciben un valor de dos, si se presentan de } \\
\text { forma ocasional un valor de uno, o si nunca } \\
\text { se manifiestan se asigna un valor de cero. } \\
F A I=F A Q 1+F A Q 2 ; F A I \in[0,4]\end{array}$ & $\begin{array}{l}\text { Propuesta del } \\
\text { autor con base en } \\
\text { Cucinelli et al. } \\
\text { (2019) }\end{array}$ \\
\hline $\begin{array}{l}\text { Índice de } \\
\text { Comporta- } \\
\text { miento } \\
\text { Financiero } \\
(F B I)\end{array}$ & $\begin{array}{l}\text { Variable ordinal que evalúa la planeación } \\
\text { financiera y el cumplimiento de compromisos } \\
\text { financieros. Corresponde a la suma de cinco } \\
\text { ítems binarios. } \\
F B I=F B Q 1+F B Q 2+F B Q 3+F B Q 4+F B Q 5 \text {; } \\
F B I \in[0,5]\end{array}$ & $\begin{array}{l}\text { Propuesta del } \\
\text { autor con base en } \\
\text { Cucinelli et al. } \\
\text { (2019); Villagó- } \\
\text { mez y González } \\
(2014)\end{array}$ \\
\hline \multicolumn{3}{|c|}{ Variables independientes } \\
\hline Género (Gen) & $\begin{array}{l}\text { Variable binaria: uno para femenino, cero } \\
\text { para masculino }\end{array}$ & $\begin{array}{l}\text { Almenberg y } \\
\text { Widmark (2011); }\end{array}$ \\
\hline Edad (Edad) & $\begin{array}{l}\text { Variable ordinal: en el modelo econométrico } \\
\text { se ha analizado como número entero positivo } \\
\text { que fluctúa entre } 18 \text { y } 70 \text {; para fines de } \\
\text { estadística descriptiva se agrupó en nueve } \\
\text { cohortes. }\end{array}$ & $\begin{array}{l}\text { Boisclair et al. } \\
\text { (2017); Cucinelli } \\
\text { et al. }(2019) \text {; } \\
\text { Klapper } \text { et al. } \\
\text { (2015); Potrich et } \\
\text { al. (2015); Van }\end{array}$ \\
\hline $\begin{array}{l}\text { Nivel educativo } \\
(E d u c)\end{array}$ & $\begin{array}{l}\text { Variable ordinal, significa el máximo grado de } \\
\text { estudios: sin educación formal (0), preescolar } \\
\text { o primaria (1), secundaria (2), preparatoria } \\
\text { (3), universidad o equivalente (4) y estudios } \\
\text { de posgrado (5). }\end{array}$ & Rooij et al. (2011) \\
\hline $\begin{array}{l}\text { Nivel de } \\
\text { ingreso } \\
\text { (Ingr) }\end{array}$ & $\begin{array}{l}\text { Variable entera medida en miles de pesos por } \\
\text { año. Se clasificó en nueve cohortes: sin } \\
\text { ingresos }(0) \text {, ingresos mayores que cero y } \\
\text { hasta } 40(1) \text {, más de } 40 \text { y hasta } 60 \text { (2), más de } \\
60 \text { y hasta } 80 \text { (3) y así sucesivamente de } 20 \text { en } \\
20 \text {, hasta llegar a más de } 160 \text { (8). Si no } \\
\text { reportaron ingresos, el registro no se incluyó } \\
\text { en el análisis. }\end{array}$ & $\begin{array}{l}\text { Almenberg y } \\
\text { Widmark (2011); } \\
\text { Klapper } \text { et al. } \\
\text { (2015); Potrich et } \\
\text { al. }(2015) \text {; Van } \\
\text { Rooij et al. (2011) }\end{array}$ \\
\hline
\end{tabular}


Cuadro 3 (continuación)

Variable

Estado civil $(E s t C)$
Descripción

Variable binaria: uno para personas casadas o que viven con su pareja; cero para individuos solteros, nunca antes casados, viudos, divorciados o separados.
Fuente

Cucinelli et al. (2019); Van Rooij et al. (2011)

Variables de control

Tamaño de la Variable ordinal: tres si la localidad tiene 100 localidad mil habitantes o más; dos si está entre $15 \mathrm{mil}$

Cucinelli et al. (TLoc) y 99,999; uno si tiene de 2500 a 14,999 y

(2019) cero si tiene menos de 2500 .

Fuente: elaboración propia con datos de las fuentes que en el cuadro se indican.

$$
\begin{gathered}
\text { logityk }=\alpha_{k}+\beta_{1} \cdot \operatorname{Gen}_{i j}+\beta_{2} \cdot \operatorname{Edad}_{i j}+\beta_{3} \cdot E d u c_{i j}+\beta_{4} \cdot \operatorname{Ingr}_{i j}+\beta_{5} \cdot E s t C_{i j}+(4) \\
\beta_{6} \cdot T L o c_{i j}+e_{i j}
\end{gathered}
$$

Así, $\gamma_{k}$ es la probabilidad de que la persona $i$ de la región $j$ obtenga $k$ o más aciertos; $\alpha_{k}$ corresponde a los umbrales entre categorías consecutivas, es decir, $\alpha_{3}$ marca el punto en el que se pasa de obtener 2 aciertos a obtener 3 en el índice en cuestión; y $e_{i j}$ es el residual que en esta investigación se asume sigue una distribución logit con media 0 y varianza $\pi^{2} / 3$.

Para el índice de conocimiento financiero, $F K I_{i j}$, y el conjunto $X_{\mathrm{i}}$ de características de la persona $i$, se establecen las ecuaciones (5) y (6):

$$
\begin{aligned}
& \operatorname{logit}_{K}\left(\gamma_{k}\right)=\operatorname{logit}\left\{\operatorname{Pr}\left(F K I_{i j} \geq k \mid X_{i j}\right)\right\} \equiv \log \left\{\frac{\operatorname{PrFKI}_{i j} \geq k}{1-\operatorname{PrFKI} I_{i j} \geq k}\right\}=\log \left(\frac{\gamma_{k}}{1-y_{k}}\right) \\
& \gamma_{k}=\operatorname{Pr}\left(F K I_{i j} \geq k \mid X_{i j}\right)
\end{aligned}
$$

De manera análoga, los índices de actitud y comportamiento financiero, $F A I_{i j}$ y $F B I_{i j}$, para la persona $i$ de la región $j$, se formulan en las ecuaciones $(7)$ y $(8)$, respectivamente:

$$
\begin{gathered}
\operatorname{logit}_{A}\left(\gamma_{k}\right)=\operatorname{logit}\left\{\operatorname{Pr}\left(F A I_{i j} \geq k \mid X_{i j}\right)\right\} \equiv \log \left\{\frac{\operatorname{Pr}\left(F A I_{i j} \geq k\right)}{1-\operatorname{Pr} F A I_{i j} \geq k}\right\} \\
\operatorname{logit}_{b}\left(\gamma_{k}\right)=\operatorname{logit}\left\{\operatorname{Pr}\left(F B I_{i j} \geq k \mid X_{i j}\right)\right\} \equiv \log \left\{\frac{\operatorname{Pr}\left(F B I_{i j} \geq k\right)}{1-\operatorname{Pr} F B I_{i j} \geq k}\right\}
\end{gathered}
$$

En la siguiente sección se comentan los resultados obtenidos por índice para cada región. 


\section{Análisis y resultados}

Para cada componente del alfabetismo financiero se calcularon los promedios de aciertos y porcentajes de personas consideradas alfabetas financieras. A partir de ellos se efectuaron pruebas de análisis de varianza, con el estadístico chi $^{2}$ de Pearson, para comprobar las diferencias interregionales. Después se construyeron modelos logit ordenados, por separado para cada región, para los índices de conocimiento, actitud y comportamiento.

Con los datos de la ENIF 2018 (Inegi, 2019a), se estima que entre los mexicanos de 18 a 70 ańos el porcentaje de alfabetas en materia de conocimiento financiero es 34.9. Este resultado a nivel nacional es comparable con el consignado por Klapper et al. (2015) para economías emergentes que fluctúa entre 24 y $42 \%$, pero bajo en contraste con el observado en economías desarrolladas que va de 37 a $68 \%$. De manera similar, se estiman los índices de actitud financiera en $60.9 \%$ y comportamiento financiero en 40 por ciento. Estos resultados pueden observarse en el cuadro 4.

En México, el alfabetismo financiero presenta diferencias regionales significativas. La Ciudad de México exhibe los promedios y los porcentajes más altos en los tres índices. El nivel de conocimiento financiero en esta región, calculado en $50.8 \%$, es ligeramente superior al de países como España o Croacia (Klapper et al., 2015).

La región Sur registra el índice de conocimiento financiero más bajo, con un porcentaje de alfabetas estimado en $27.2 \%$, comparable al de países como Egipto o Irak (Klapper et al., 2015); sin embargo, esta misma región presenta el segundo porcentaje más alto de personas con actitud financiera positiva, 63.7 por ciento.

Las regiones norteñas tienen resultados similares entre sí. El Noroeste destaca por el nivel de conocimiento y el Noreste por el de comportamiento. El Noroeste registra 38.6\% de conocimiento financiero, comparable con los de Camerún o Rusia; el Noreste alcanza 33.3\%, equiparable a los registrados en Argelia o Corea del Sur (Klapper et al., 2015).

La región Occidente y Bajío tiene un porcentaje de alfabetas financieros de $34.8 \%$, bajo la perspectiva de conocimiento, comparable con los resultados de Brasil o Bulgaria (Klapper et al., 2015). Esta región presenta los menores porcentajes de actitud y comportamiento financiero, con 55.4 y $37.1 \%$, respectivamente.

La región Centro Sur y Oriente, en donde vive 30.9\% de la población del país y que aporta la quinta parte del PIB, registra un porcentaje de conocimiento financiero de $34.1 \%$, similar al de Uganda y ligeramente superior al de Belice (Klapper et al., 2015). Es la segunda con menor porcentaje de comportamiento financiero positivo y la tercera con mayor porcentaje de actitud financiera. 


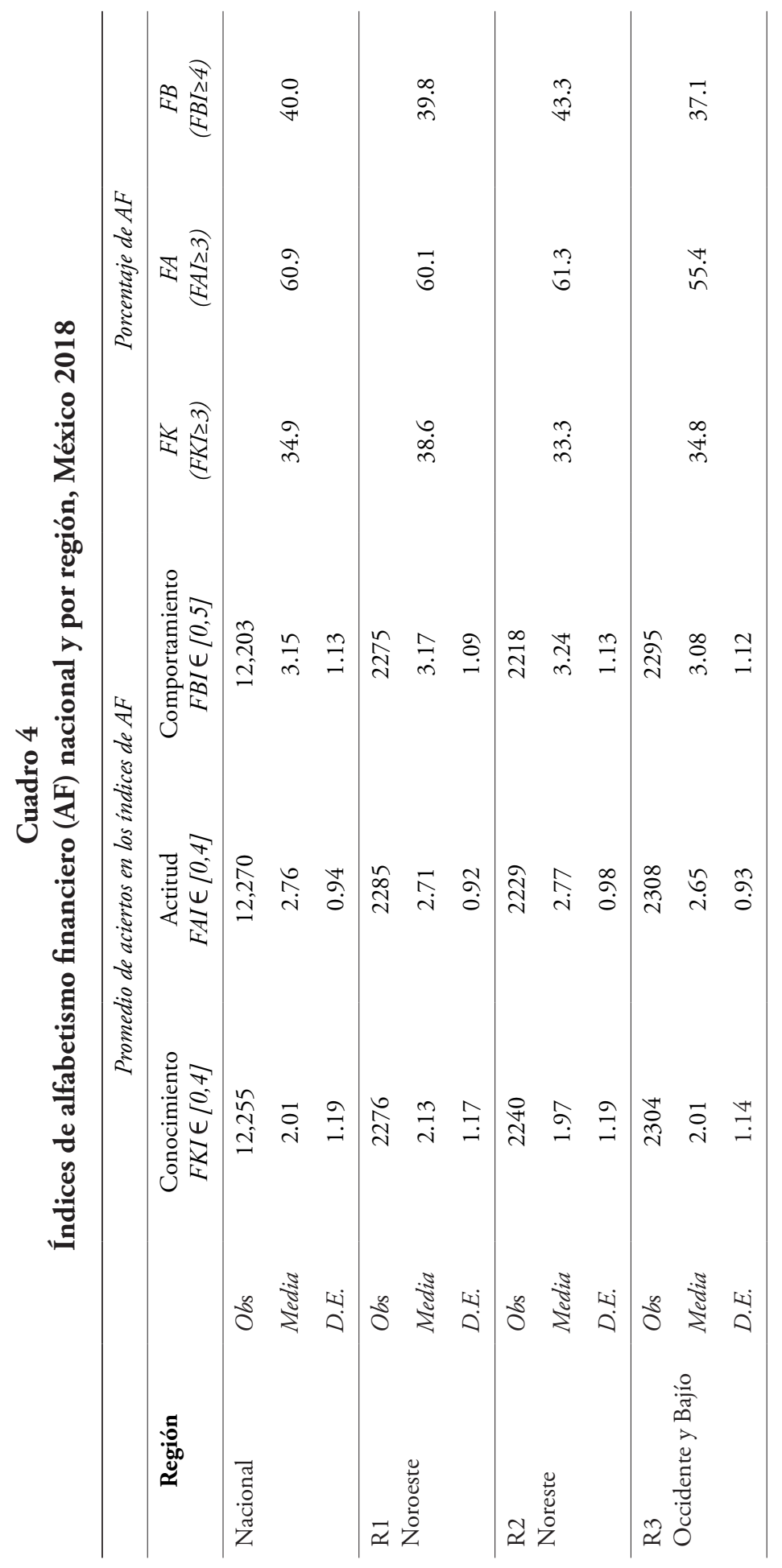




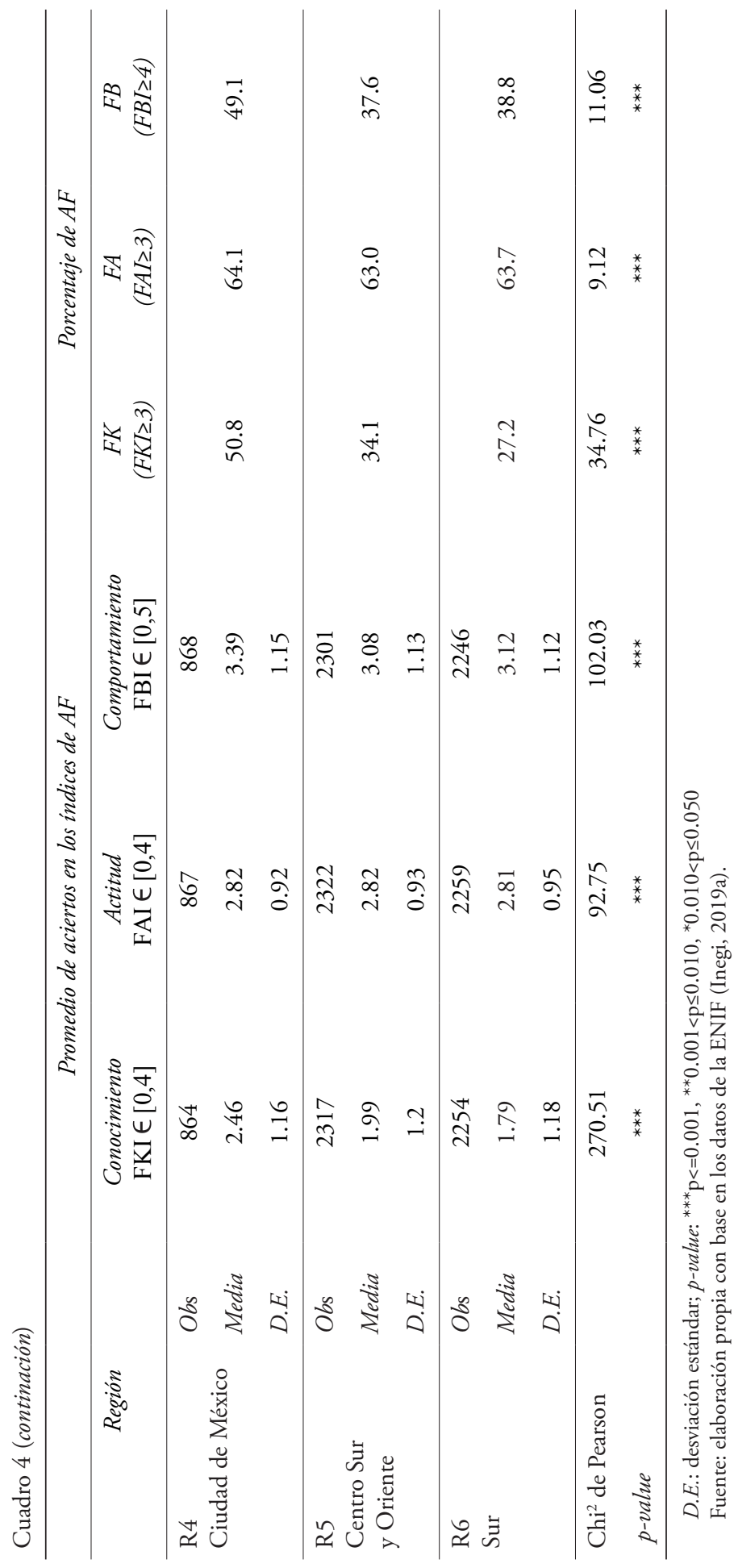


Estos datos constituyen evidencia para demostrar que $\left(\mathrm{H}_{1}\right)$ en México prevalece un nivel de conocimiento financiero bajo en todas las regiones analizadas en comparación con las naciones desarrolladas, con excepción de la Ciudad de México, según lo observado por Klapper et al. (2015).

$\mathrm{El}$ análisis de varianzas indica que existen diferencias interregionales en los índices de alfabetismo financiero debidas a factores distintos a la aleatoriedad aplicada a la muestra seleccionada (cuadro 4). Esto sugiere que un solo modelo para todas las regiones del país sería inadecuado. Por lo anterior, se pusieron a prueba algunas características socioeconómicas regionales para determinar cuáles son las que mejor explican el alfabetismo financiero de los habitantes de las seis regiones bajo estudio.

Antes de construir los modelos econométricos, se llevaron a cabo análisis de correlaciones para identificar si existe multicolinealidad entre variables dependientes e independientes. Se aplicaron las pruebas del coeficiente de Pearson con un criterio máximo de 0.50 y chi-cuadrada con un criterio máximo de 0.05 . Tras ellas se observó que ninguna variable presenta problemas de sobrerrepresentación. De manera similar, se estimaron factores de inflación de la varianza que consignaron valores aceptables $(\mathrm{VIF}<5.0)$, con un factor medio de 1.17. Por lo anterior, se considera que todas las variables propuestas son susceptibles de incluirse en los modelos regionales.

Adicionalmente se efectuó un análisis de componentes principales para determinar si es posible condensar la información contenida en la serie de variables originales. Para las observaciones a nivel nacional y los subconjuntos por región las medidas de idoneidad de Kaiser-Meyer-Olkin resultaron aceptables $(\mathrm{KMO}>0.5)$ y las pruebas de Bartlett registraron p-values menores que 0.001 , lo que confirma que existen suficientes correlaciones entre variables para llevar a cabo el análisis de factores.

En el cuadro 5 se presentan los pesos de cada variable después de realizar el análisis de factores componentes principales con rotación varimax para los datos de cada región. Se retuvieron dos factores en $R 1$ y $R 2$, $\mathrm{y}$ tres factores en el resto de las regiones. Se observa que es posible agrupar las variables en factores componentes principales, aunque estas agrupaciones varían según la región. Por ejemplo, al considerar sólo las variables con pesos mayores a 0.5 , edad y educación aparecen juntas en el mismo factor en todas las regiones; sin embargo, a ellas se puede asociar el tamaño de la localidad en $R 1$, el estado civil en $R 4$, ambas en $R 2$, o ninguna variable adicional en $R 3, R 5$ y $R G$.

A partir del análisis de factores componentes principales queda de manifiesto que las variables explicativas en esta investigación registran comportamientos distintos en cada región. De esta forma, se justifica la necesidad de construir modelos por separado, que faciliten entender esta dinámica regional para cada componente del alfabetismo financiero. 


\section{Cuadro 5}

Factores componentes principales con rotación varimax por región

\begin{tabular}{|c|c|c|c|c|c|c|c|c|c|c|c|c|c|c|c|c|c|}
\hline \multirow[t]{2}{*}{ Variable } & \multicolumn{3}{|c|}{$R 1$} & \multicolumn{2}{|r|}{$R 2$} & \multicolumn{3}{|c|}{ R3 } & \multicolumn{3}{|c|}{$R 4$} & \multicolumn{3}{|c|}{$R 5$} & \multicolumn{3}{|c|}{$R 6$} \\
\hline & $F 1$ & $F 2$ & F3 & $F 1$ & $F 2$ & F3 $F 1$ & $F 2$ & F3 & $F 1$ & $F 2$ & F3 & $F 1$ & $F 2$ & F3 & $F 1$ & F2 & F3 \\
\hline Gen & 0.1 & -0.8 & - & 0.1 & -0.8 & $\begin{array}{ll}-0.8 \\
\end{array}$ & 0.0 & -0.1 & -0.2 & -0.8 & 0.1 & -0.8 & $3-0.1$ & 0.1 & 0.1 & $1-0.8$ & $8-0.1$ \\
\hline Edad & -0.6 & 0.1 & - & -0.6 & 0.1 & -0.0 & 0.1 & 0.9 & 0.7 & -0.1 & 0.3 & 0.0 & 0.9 & 0.1 & 0.1 & 10.0 & $\begin{array}{ll}0.9 \\
0.9\end{array}$ \\
\hline Educ & 0.8 & 30.1 & - & 0.8 & 0.2 & 0.2 & 0.5 & -0.6 & -0.6 & 0.4 & 0.2 & 0.3 & $3-0.6$ & 0.5 & 0.5 & $\begin{array}{ll}5 & 0.2\end{array}$ & $2-0.6$ \\
\hline Ingr & 0.3 & 0.8 & - & 0.3 & 0.8 & $-\quad 0.8$ & 0.2 & -0.2 & -0.3 & 0.7 & 0.2 & 0.8 & -0.2 & 0.2 & 0.3 & $\begin{array}{ll}3 & 0.8\end{array}$ & $8-0.2$ \\
\hline EdoC & -0.3 & 0.2 & - & -0.5 & 0.2 & 0.2 & -0.5 & 0.2 & 0.6 & 0.3 & -0.3 & 0.2 & 20.3 & -0.4 & -0.6 & $\begin{array}{ll}6 & 0.3\end{array}$ & $\begin{array}{ll}3 & 0.0\end{array}$ \\
\hline TLoc & 0.6 & 0.1 & - & 0.5 & 0.2 & 0.1 & 0.9 & 0.1 & 0.0 & 0.1 & 0.9 & 0.0 & 0.1 & 0.9 & 0.8 & $\begin{array}{ll}3 & 0.2\end{array}$ & 20.0 \\
\hline $\begin{array}{l}\text { Fact. } \\
\text { Ret. }\end{array}$ & & 2 & & & 2 & & 3 & & & 3 & & & 3 & & & 3 & \\
\hline $\begin{array}{l}\text { Var. } \\
\text { Expl. }\end{array}$ & & 0.5 & & & 0.5 & & 0.7 & & & 0.6 & & & 0.7 & & & 0.7 & \\
\hline Obs. & & 2197 & & & 2140 & & 2196 & & & 708 & & & 2250 & & & 2230 & \\
\hline
\end{tabular}

Fuente: elaboración propia con datos de la ENIF (Inegi, 2019a) y Stata (StataCorp, 2017).

\subsection{Conocimiento financiero}

En todas las regiones de México, el conocimiento financiero está estrechamente relacionado con el nivel educativo de las personas entre $18 \mathrm{y}$ 70 ańos. Entre mayor es el grado de estudios, las probabilidades de comprender mejor los conocimientos financieros son más altas. En contraste, en ninguna región se encontraron evidencias para afirmar que el estado civil está relacionado con el conocimiento financiero. Estos resultados pueden observarse en el cuadro 6 .

Por otra parte, la edad, el género, el tamaño de la localidad y el nivel de ingreso afectan de manera distinta al conocimiento financiero en cada región. Un patrón similar se aprecia en las regiones Noroeste (R1) y Noreste (R2). Sus modelos incluyen con niveles altos de significancia, además del nivel educativo, el ingreso y el tamaño de la localidad. En éstas, si una persona habita en una localidad altamente poblada y entre mayor es su ingreso, es más probable que su desempeño sea mejor en el índice de conocimiento financiero. En la región Centro Sur y Oriente (R5), se aprecia un patrón similar al de las regiones del norte, pero en ésta la edad también tiene un papel significativo. Entre los jóvenes hay mayor predisposición al conocimiento financiero. En las regiones Occidente y Bajío (R3) y Sur (R5) las variables de mayor peso en la estimación del conocimiento financiero, además de la educación, son el tamaño de la 


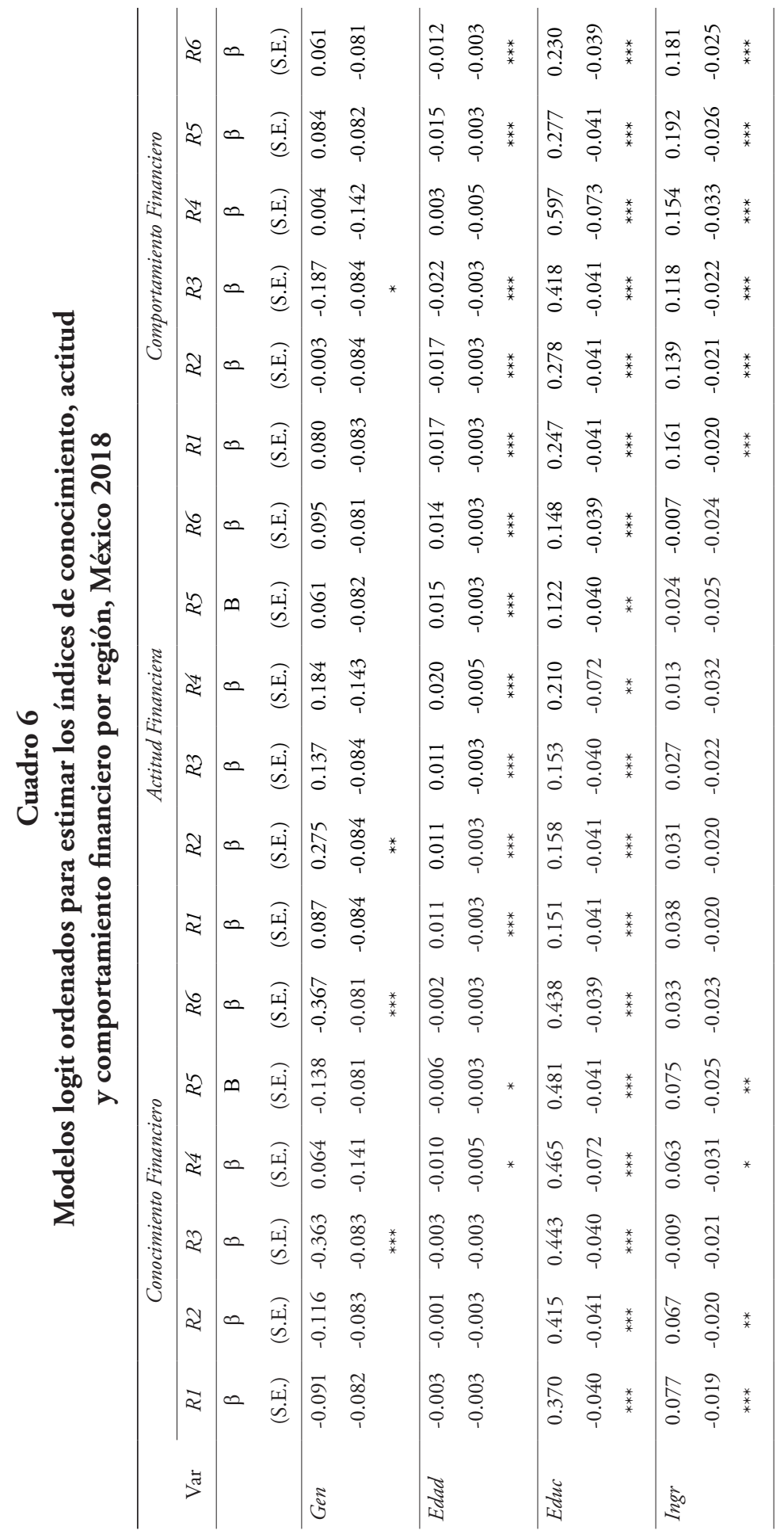




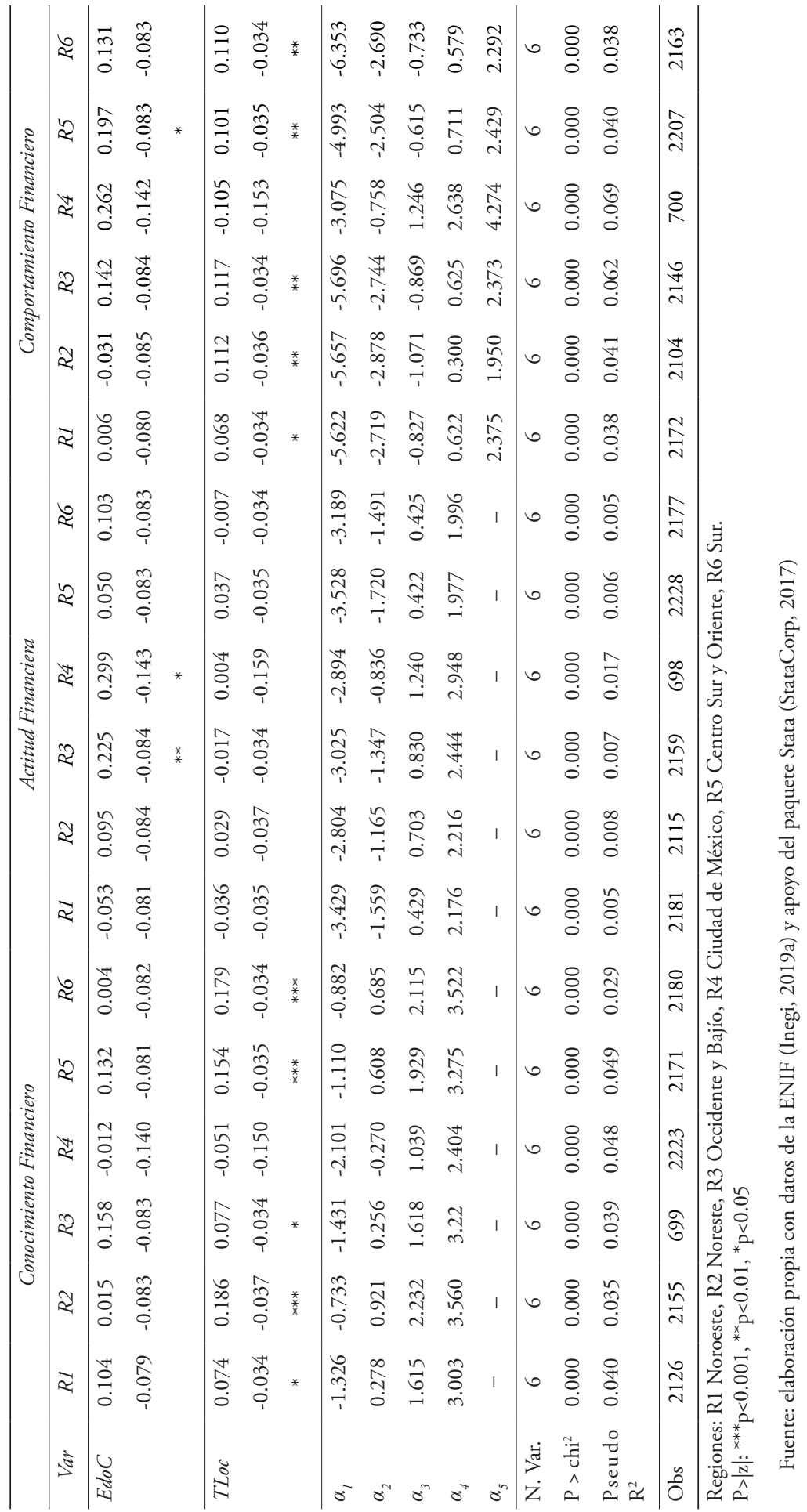


localidad y el género. Es decir, en éstas es esencial educar financieramente a las mujeres en las localidades rurales para aminorar la brecha observada.

En la Ciudad de México (R4) el nivel educativo es principalmente el que determina una buena estimación del conocimiento financiero y en menor medida la edad y el nivel de ingreso. En especial se recomienda atender la educación de los adultos mayores que ganan menos.

Por ejemplo, la probabilidad de que una persona sea alfabeta en términos de conocimiento financiero en la región Noreste, $j=2$ puede obtenerse al sustituir en la ecuación (4) los valores obtenidos con el modelo logit para esta región, presentados en el cuadro 6 , con $k=3$ aciertos y $e_{i j}=0$. La expresión correspondiente queda de la siguiente forma:

$$
\begin{gathered}
\operatorname{logit}_{K}\left(\gamma_{3}\right)=-2.232-0.116 \cdot \text { Gen }_{i 2}-0.001 \cdot \text { Edad }_{i 2}+0.415 \\
\text { Educ }_{i 2}+0.067 \cdot \text { Ingr }_{i 2}+0.015 \cdot \text { EstC }_{i 2}+0.186 \cdot \text { TLoc }_{i 2}
\end{gathered}
$$

Al sustituir valores para una mujer $\left(\operatorname{Gen}_{i 2}=1\right)$, de 24 años $\left(\operatorname{Edad}_{i 2}=24\right)$, casada $\left(E d o C_{i 2}=1\right)$, con un nivel de escolaridad máximo de primaria $\left(E d u c_{i 2}=2\right)$, que gana 48,000 pesos al año $\left(\operatorname{Ingr}_{i 2}=2\right)$ y que vive en una localidad de 11,500 habitantes $\left(\right.$ TLOC $\left._{i 2}=1\right)$, a partir de las ecuaciones (6) y (9) se estima $\operatorname{logit}_{K}\left(\gamma_{3}\right)=-1.622$. De este resultado y la ecuación (5) se desprende que la probabilidad de que esta persona sea alfabeta financiera, en esta región, es 0.165 , como se muestra en la ecuación (10).

$$
\operatorname{Pr}\left(F K I_{i 2} \geq 3\right)=\gamma_{3 \text { (primaria) }}=0.165
$$

Si esta persona hubiera terminado preparatoria, con exactamente las mismas características restantes, la probabilidad de que fuera alfabeta en términos de conocimiento financiero, como se presenta en la ecuación (11), sería casi el doble.

$$
\operatorname{Pr}\left(F K I_{i 2} \geq 3\right)=\gamma_{3 \text { (preparatoria })}=0.312
$$

A nivel nacional es esencial elevar el nivel educativo para aspirar a mejorar los resultados en el índice de conocimiento financiero. Como lo mencionaron Villagómez e Hidalgo (2017), la educación formal y en especial las habilidades matemáticas son críticas en esta tarea. A nivel regional es necesario reducir la desigualdad en las oportunidades educativas. Específicamente en las regiones Noroeste (R1), Noreste (R2) y Centro Sur y Oriente (R5) se recomienda fortalecer la educación financiera de las familias con menores ingresos. En las regiones Occidente y Bajío (R3) y Sur (R6) la focalización de estrategias debe considerar mitigar la brecha de género. 


\subsection{Actitud financiera}

La actitud financiera de los mexicanos, su predisposición a decidir y actuar en materia financiera, está relacionada con la edad y el nivel educativo. El índice de actitud financiera registra mejores resultados entre personas mayores que en jóvenes, y entre individuos con mayor grado de escolaridad. Estas dos variables son significativas en todas las regiones, resultado consistente con el obtenido por Finke et al. (2016), quienes observaron que a mayor edad, las personas desarrollan mayor confianza en la administración del dinero, así como en el propio conocimiento financiero.

En la región Noreste (R2) se aprecia además que las mujeres muestran una actitud más positiva que los hombres. En las regiones Occidente y Bajío (R3) y Ciudad de México (R4) los resultados son mejores entre quienes están casados o viven con su pareja. Es interesante notar que la actitud financiera no depende del nivel de ingreso o del tamaño de la localidad, es decir, la oferta de servicios financieros que provee una ciudad muy poblada o a la que pudieran tener acceso sólo las personas de nivel económico alto, no influyen en la actitud financiera de los mexicanos.

Con los valores obtenidos en el modelo logit ordenado, presentados en el cuadro 6 , se puede construir a partir de la ecuación (4) una expresión para la región $j=2$, con $k=3$ aciertos y $e_{i j}=0$ :

$$
\begin{aligned}
& \operatorname{logit}_{A}\left(\gamma_{3}\right)=-0.703+0.275 \cdot \text { Gen }_{i 2}+0.011 \cdot \operatorname{Edad}_{i 2}+0.158 \text {. } \\
& E_{\text {duc }}+0.031 \cdot \text { Ingr }_{i 2}+0.095 \cdot \text { Est }_{i 2}+0.029 \cdot \text { TLoc }_{i 2}
\end{aligned}
$$

$\mathrm{Al}$ sustituir en la ecuación (12) los valores para una mujer $\left(\operatorname{Gen}_{i 2}=1\right)$, de 24 años $\left(E_{d a d}=24\right)$, casada $\left(E d o C_{i 2}=1\right)$, con un nivel de escolaridad máximo de secundaria $\left(E d u c_{i 2}=2\right)$, que gana 48,000 pesos al año $\left(\operatorname{Ingr}_{i 2}=2\right)$ y que vive en una localidad de 11,500 habitantes $\left(T L o c_{i 2}=1\right)$, se estima $\operatorname{logit}_{A}\left(\gamma_{3}\right)=0.338$. Con este resultado y la ecuación (7) se obtiene que la probabilidad de que esta persona sea alfabeta bajo la perspectiva de actitud financiera es 0.584 , como se aprecia en la ecuación (13).

$$
\operatorname{Pr}\left(F A I_{i 2} \geq 3\right)=\gamma_{3(\text { Edad }=24)}=0.584
$$

Si se conservaran las mismas características socioeconómicas para esta persona, excepto que la edad fuera 64 años $\left(E_{d a d}{ }_{i 2}=64\right)$, entonces la probabilidad de considerarla alfabeta en términos de actitud financiera aumentaría más de 10 puntos porcentuales, como se presenta en la ecuación (14). 


$$
\operatorname{Pr}\left(F A I_{i 2} \geq 3\right)=\gamma_{3(\text { Edad }=64)}=0.685
$$

Las políticas de inclusión financiera deberían considerar a la educación escolar como plataforma para mejorar el desempeño en actitud financiera y en cierta forma propiciar su fortalecimiento a través de la educación intergeneracional (Benton y Keister, 2017; Lissington y Matthews, 2012). Se recomienda investigar más sobre el efecto regional que tienen otras variables, por ejemplo, en el Noreste (R2), donde las mujeres presentan una actitud financiera más positiva que los hombres, y en las regiones Occidente y Bajío (R3) y Ciudad de México (R4), donde estar casado contribuye significativamente a fortalecer esta actitud.

\subsection{Comportamiento financiero}

En México, el nivel educativo y el ingreso guardan una relación positiva y significativa con el índice de comportamiento financiero en todas las regiones. A excepción de la Ciudad de México, la edad y el tamaño de la localidad son otros factores significativos para estimar el comportamiento financiero en el país. A menor edad y mayor tamaño de la localidad, más altas son las probabilidades de que la persona obtenga mejores resultados. Además de las cuatro variables anteriores, en la región Occidente y Bajío (R3), el género se asocia negativamente a este indicador, a favor de los hombres; y en la región Centro Sur y Oriente (R5), el estado civil es también significativo, a favor de los casados.

$\mathrm{Al}$ sustituir en la ecuación (4) los valores obtenidos con el modelo logit ordenado, presentados en el cuadro 6 , se encuentra una expresión para la región $j=1$, con $k=4$ aciertos, y $e_{i j}=0$ :

$$
\begin{gathered}
\operatorname{logit}_{B}\left(\gamma_{4}\right)=-0.622+0.080 \cdot \text { Gen }_{i 1}-0.017 \cdot \text { Edad }_{i 1}+0.247 \\
\text { Educ }_{i 1}+0.161 \cdot \text { Ingr }_{i 1}+0.006 \cdot \text { EstC }_{i 1}+0.068 \cdot \text { TLoc }_{i 1}
\end{gathered}
$$

$\mathrm{Al}$ probar en la ecuación (15), los valores para una mujer $\left(\mathrm{Gen}_{i 1}=1\right)$, de 42 años $\left(E_{d a d_{i 1}}=42\right)$, casada $\left(E d o C_{i 1}=1\right)$, con un nivel de escolaridad máximo de secundaria $\left(E d u c_{i 1}=2\right)$, que gana 48,000 pesos al año $\left(\operatorname{Ingr}_{i 1}=2\right)$ y que vive en una localidad de más de 100,000 habitantes $\left(T L o c_{i 1}=3\right)$, se estima $\operatorname{logit}_{B}\left(\gamma_{4}\right)=-0.230$. Con este resultado y la ecuación (8) se pronostica que esta persona obtendría cuatro o más aciertos en el índice de comportamiento financiero con una probabilidad de 0.443 , como se muestra en la ecuación (16).

$$
\operatorname{Pr}\left(F B I_{i 1} \geq 4\right)=\gamma_{4(\text { Región }=1)}=0.443
$$


Al comparar este resultado con la estimación para una persona de las mismas características socioeconómicas, pero que vive en la Ciudad de México $(j=4)$, su probabilidad de considerarla alfabeta en términos de comportamiento financiero sería significativamente distinta, como se aprecia en la ecuación (17).

$$
\operatorname{Pr}\left(F B I_{i 4} \geq 4\right)=\gamma_{4(\text { Región }=4)}=0.258
$$

Para validar estos resultados, se revisaron las observaciones obtenidas de la ENIF 2018 (Inegi, 2019a). Se encontró que en el Noroeste (R1) hay 135 mujeres, con secundaria como máximo grado de estudios, casadas, de cualquier edad (promedio 40.5) y que habitan en una localidad de más de 100 mil habitantes, mientras que en la Ciudad de México (R4) se registraron 49 personas con estas características (edad promedio 42.5). De ellas, en el Noroeste (R1), 43.0\% obtuvo cuatro aciertos o más en el índice de comportamiento financiero, en tanto que en la Ciudad de México (R4), este indicador es de $22.4 \%$. Es decir, el modelo logit ordenado se aproxima a los datos observados con un nivel de precisión aceptable.

Fernandes et al. (2014) argumentaron que la educación financiera si bien contribuye a fortalecer el conocimiento, al mismo tiempo debe reforzar comportamientos financieros deseables, por ejemplo, el hábito de elaborar y apegarse a un presupuesto. A nivel nacional, es necesario insistir en elevar el nivel educativo para aspirar a obtener mejores resultados en el índice de comportamiento financiero. A nivel regional, es importante adecuar las estrategias derivadas de la política de inclusión financiera a las diferentes características locales. Además del nivel educativo y el ingreso, fuera de la Ciudad de México (R4) es necesario dirigir los esfuerzos a las comunidades rurales y los adultos mayores. En la región Occidente y Bajío (R3) debe procurarse la reducción de brechas en beneficio de las mujeres que residen en localidades rurales. En la región Centro Sur y Oriente (R5) es recomendable establecer estrategias educativas focalizadas en la gente con ingresos bajos, localidades poco pobladas y aquellos que no tienen cónyuge.

Por todo lo anterior, se puede afirmar que existen elementos para comprobar que $\left(\mathrm{H}_{2}\right)$ los determinantes socioeconómicos del alfabetismo financiero varían de región a región, en cada uno de sus tres componentes: conocimiento, actitud y comportamiento. Solamente el nivel educativo es el único factor asociado de forma positiva a los tres componentes en todas las regiones de México. Este resultado es consistente con lo observado por Antonio-Anderson et al. (2020), quienes reconocieron a la educación como un factor determinante del alfabetismo financiero en todo el país, aunque en su investigación no diferenciaron por componente. 


\section{Conclusiones}

El objetivo de esta investigación ha sido medir el alfabetismo financiero en México y analizar sus determinantes con un enfoque regional, a partir de los datos de la Encuesta Nacional de Inclusión Financiera, ENIF (Inegi, 2019a). El nivel de alfabetismo financiero, por componente, se estimó en $40.0 \%$ para comportamiento financiero, $60.9 \%$ para actitud financiera y $34.9 \%$ para conocimiento financiero. Este último es equiparable al nivel observado en países emergentes, pero bajo en comparación con naciones desarrolladas, tal como lo consignaron Klapper et al. (2015).

En respuesta a las preguntas planteadas en esta investigación, se puede afirmar que la educación es el único factor asociado de forma directa a los niveles de conocimiento, actitud y comportamiento financieros en todas las regiones de México y que la relevancia del resto de los factores socioeconómicos analizados, género, edad, ingreso, estado civil y tamaño de la localidad varía de región a región, de manera similar a lo observado por Cucinelli et al. (2019) en Italia.

En México persiste una brecha de género a favor de los hombres en conocimiento y comportamiento financiero. Ésta no es tan notoria en la actitud, en cuyo caso favorecería a las mujeres. Asimismo, la política de inclusión financiera debería de considerar la brecha en los resultados obtenidos por los habitantes de localidades altamente pobladas en contraste con los de comunidades rurales. Como lo observaron Cucinelli et al. (2019), éstas ofrecen mejor acceso a la educación, más servicios financieros y tecnologías de la información.

A pesar de los avances de las últimas décadas en materia de educación en México, los niveles de desigualdad y exclusión financiera siguen siendo altos (García et al., 2013). A nivel regional, es necesario ampliar la cobertura de los servicios educativos y financieros en las localidades menos pobladas. Los programas de educación financiera son un elemento crítico en la instrumentación de estrategias de reducción de la pobreza; éstos deben considerar los factores estructurales asociados a las comunidades y tender al fortalecimiento del capital social (García et al., 2013). Se recomienda investigar más sobre los posibles fallos del modelo de educación financiera actual, que traslada toda la responsabilidad de las decisiones al consumidor, como lo advierte Willis (2008), al referirse a cómo la educación es necesaria pero no suficiente para aspirar a un mayor bienestar financiero.

Esta investigación aporta evidencias que explican que el alfabetismo financiero está determinado por factores socioeconómicos distintos para cada región de México. Entre sus principales limitaciones se encuentra la falta de datos para realizar un análisis longitudinal que permita observar 
cambios en las condiciones del entorno y su impacto en el nivel de alfabetismo financiero de la población. Sus hallazgos justifican la necesidad de diseñar estrategias de formación financiera adecuadas a los contextos regionales y locales. La instrumentación apropiada de estrategias facilitará la mitigación de brechas en el alfabetismo financiero y la inclusión financiera. Una población mejor preparada financieramente suele alcanzar mayores niveles de bienestar en menor tiempo, contribuye a dinamizar la economía y a mejorar el desarrollo económico y social.

\section{Fuentes consultadas}

Almenberg, Johan y Widmark, Olof (2011), "Numeracy, Financial Literacy and Participation in Asset Market", Rochester, SSRN (Social Science Research Network), <https://bit.ly/3oN90Ir>, 19 de septiembre de 2020.

Altman, Morris (2012), "Implications of behavioural economics for financial literacy and public policy", The Journal of Socio-Economics, 41 (5), Ámsterdam, Elsevier, pp. 677-690, doi:10.1016/j. socec.2012.06.002

Antonio-Anderson, Ceyla; Peña Càrdenas, Maricela Carolina y López Saldaña, Cristina del Pilar (2020), "Determinantes de la alfabetización financiera”, Investigación Administrativa, 49 (125), Ciudad de México, Instituto Politécnico Nacional, pp. 1-16, <https:// bit.ly/2JW0pVd>, 19 de septiembre de 2020.

Arceo-Gomez, Eva Olimpia y Villagómez, Alejandro (2017), "Financial literacy among Mexican high school teenagers", International Review of Economics Education, 24 (1), Ámsterdam, Elsevier, pp. 1-17, doi:10.1016/j.iree.2016.10.001

Atkinson, Adele y Messy, Flore Anne (2012), "OECD Working Papers on Finance, Insurance and Private Pensions No. 15. Measuring Financial Literacy: Results of the OECD/International Network on Financial Education (INFE) Pilot Study", París, OCDE (Organización para la Cooperación y el Desarrollo Económicos), doi:10.1787/5k9csfs90fr4-en

Baglioni, Angelo; Colombo, Luca y Piccirilli, Giulio (2018), "On the Anatomy of Financial Literacy in Italy”, Economic Notes, 47 (2-3), 
Hoboken, Wiley Online Library, pp. 245-304, doi:10.1111/ ecno. 12111

Bay, Charlotta; Catasús, Bino y Johed, Gustav (2014), "Situating financial literacy", Critical Perspectives on Accounting, 25 (1), Ámsterdam, Elsevier, pp. 36-45, doi:10.1016/j.cpa.2012.11.011

Benton, Richard y Keister, Lisa (2017), "The lasting effect of intergenerational wealth transfers: Human capital, family formation, and wealth", Social Science Research, vol. 68, Ámsterdam, Elsevier, pp.1-14, doi:10.1016/j.ssresearch.2017.09.006

Bernheim, Douglas; Garrett, Daniel y Maki, Dean (2001), "Education and saving: The long-term effects of high school financial curriculum mandates", Journal of Public Economics, 80 (3), Ámsterdam, Elsevier, pp. 435-465, doi:10.1016/S0047-2727(00)00120-1

Boisclair, David; Lusardi, Annamaria y Michaud, Pierre-Carl (2017), "Financial literacy and retirement planning in Canada", Journal of Pension Economics and Finance, 16 (3), Cambridge, Cambridge University Press, pp. 277-296, doi:10.1017/S1474747215000311

Carpena, Fenella; Cole, Shawn; Shapiro, Jeremy y Zia, Bilal (2017), "The ABCs of Financial Education: Experimental Evidence on Attitudes, Behavior, and Cognitive Biases", Management Science, 65 (1), Catonsville, INFORMS (Institute for Operations Research and the Management Sciences), pp. 346-369, doi:10.1287/ mnsc.2017.2819

Clark, Robert; D’Ambrosio, Madeleine; McDermed, Ann y Sawant, Kshama (2006), "Retirement plans and saving decisions: The role of information and education", Journal of Pension Economics and Finance, 5 (1), Cambridge, Cambridge University Press, pp. 45-67, doi:10.1017/S1474747205002271

CNBV (Comisión Nacional Bancaria y de Valores) (2020), "Política Nacional de Inclusión Financiera", Ciudad de México, CNBV, <https://bit.ly/3a2xSYT>, 19 de septiembre de 2020.

Conapo (Consejo Nacional de Población) (2017), "Proyecciones de la Población de México y de las entidades federativas, 2016-2050", Ciudad de México, Conapo, <https://bit.ly/3oMTJHV>, 19 de septiembre de 2020 . 
Coneval (Consejo Nacional de Evaluación de la Política de Desarrollo Social) (2019), "Medición de la pobreza. Pobreza en México", Ciudad de México, Coneval, <https://bit.ly/3oBfoTg>, 2 de abril de 2020

Cucinelli, Doriana; Trivellato, Paolo y Zenga, Mariangela (2019), "Financial Literacy: The Role of the Local Context", Journal of Consumer Affairs, 53 (4), Hoboken, Wiley Online Library, pp. 1874-1919, doi:10.1111/joca.12270

Cude, Brenda (2010), "Financial Literacy 501", Journal of Consumer Affairs, 44 (2), Hoboken, Wiley Online Library, pp. 271-275, doi:10.1111/j.1745-6606.2010.01168.x

Ćumurović, Aida y Hyll, Walter (2019), "Financial Literacy and SelfEmployment", Journal of Consumer Affairs, 53 (2), Hoboken, Wiley Online Library, pp. 455-487, doi:10.1111/joca.12198

Das, Sanjib (2016), "Financial literacy: measurement and determinants", International Journal of Economic and Business Review, 4 (6), Tiruchirapalli, EPRA Journals, pp. 88-93, <https://bit. ly/37SmtrT>, 1 de octubre de 2020.

Fernandes, Daniel; Lynch, John y Netemeyer, Richard (2014), "Financial Literacy, Financial Education, and Downstream Financial Behaviors", Management Science, 60 (8), Catonsville, INFORMS (Institute for Operations Research and the Management Sciences), pp. 1861-1883, doi:10.1287/mnsc.2013.1849

Finke, Michael; Howe, John y Huston, Sandra (2016), "Old Age and the Decline in Financial Literacy”, Management Science, 63 (1), Catonsville, INFORMS (Institute for Operations Research and the Management Sciences), pp. 213-230, doi:10.1287/ mnsc. 2015.2293

García, Nidia; Grifoni, Andrea; López, Juan Carlos y Mejía, Diana (2013), "Financial Education in Latin America and the Caribbean: Rationale, Overview and Way Forward", documento de trabajo núm. 33, París, OCDE.

Huston, Sandra (2010), "Measuring Financial Literacy", Journal of Consumer Affairs, 44 (2), Hoboken, Wiley Online Library, pp. 296316, doi:10.1111/j.1745-6606.2010.01170.x 
Inegi (Instituto Nacional de Estadística y Geografía) (2019a), "Encuesta Nacional de Inclusión Financiera (ENIF) 2018”, Aguascalientes, Inegi, <https://bit.ly/377LsYP>, 19 de septiembre de 2020.

Inegi (Instituto Nacional de Estadística y Geografía) (2019b), "Encuesta Nacional de Ocupación y Empleo (ENOE), población de 15 años y más de edad", Aguascalientes, Inegi, <https://bit.ly/2W5SHKD>, 4 de diciembre de 2020 .

Inegi (Instituto Nacional de Estadística y Geografía) (2018), "PIB y cuentas nacionales", Aguascalientes, Inegi, <https://bit. ly/3n $5 \mathrm{KwKd}>, 1$ de octubre de 2020.

Inegi (Instituto Nacional de Estadística y Geografía) (2015), "Población de México", Aguascalientes, Inegi, <https://bit.ly/2JZ9f4t>, 1 de octubre de 2020.

Klapper, Leora; Lusardi, Annamaria y Panos, Georgios (2013), “Financial literacy and its consequences: Evidence from Russia during the financial crisis", Journal of Banking and Finance, 37 (10), Ámsterdam, Elsevier, pp. 3904-3923, doi:10.1016/j. jbankfin.2013.07.014

Klapper, Leora; Lusardi, Annamaria y van Oudheusden, Peter (2015), "Financial Literacy Around the World: Insights From the Standard \& Poor's Ratings Services Global Financial Literacy Survey", Washington, Global Financial Literacy Excellence Center (GFLEC), pp. 1-27, <https://bit.ly/3qNO7Pn>, 2 de abril de 2020.

Leckie, George; Morris, Tim y Steele, Fiona (2016), "Single-level and Multilevel Models for Ordinal Responses - Stata Practical”, Bristol, Centre for Multilevel Modelling, pp. 1-44, <https://bit. ly/3gEZTXy>, 4 de diciembre de 2020.

Lissington, Robert y Matthews, Claire (2012), "Intergenerational Transfer of Financial Literacy", Social Science Research Network Electronic Journal, Rochester, SSRN (Social Science Research Network), pp.1-32, doi:10.2139/ssrn.2256791

Lusardi, Annamaria y Mitchell, Olivia (2011a), "Financial Literacy and Planning: Implications for Retirement Wellbeing”, documento de trabajo núm. 17078, Cambridge, NBER (National Bureau 
of Economic Research), <https://bit.ly/3444RIy>, 1 de octubre de 2020.

Lusardi, Annamaria y Mitchell, Olivia (2011b), "Financial literacy around the world: An overview", Journal of Pension Economics and Finance, 10 (4), Cambridge, Cambridge University Press, pp. 497-508, doi:10.1017/S1474747211000448

OCDE/INFE (Organización para la Cooperación y el Desarrollo Económicos/International Network on Financial Education) (2011), "Measuring Financial Literacy: Questionnaire and Guidance Notes for Conducting an Internationally Comparable Survey of Financial Literacy", París, OCDE, <https://bit.ly/2KljQH2>, 19 de septiembre de 2020.

Potrich, Ani Caroline; Vieira, Kelmara y Kirch, Guilherme (2015), "Determinants of financial literacy: Analysis of the influence of socioeconomic and demographic variables", Revista Contabilidade \& Finanças, 26 (69), São Paulo, Universidad de São Paulo, pp. 362-377, doi:10.1590/1808-057x201501040

Remund, David (2010), "Financial literacy explicated: The case for a clearer definition in an increasingly complex economy", Journal of Consumer Affairs, 44 (2), Hoboken, Wiley Online Library, pp. 276-295, doi:10.1111/j.1745-6606.2010.01169.x

StataCorp (2017), "Stata”, [software], versión 14.2 para Windows, College Station, StataCorp LLC.

Van Rooij, Maarten; Lusardi, Annamaria y Alessie, Rob (2011), "Financial literacy and retirement planning in the Netherlands", Journal of Economic Psychology, 32 (4), Ámsterdam, Elsevier, pp. 593-608, doi:10.1016/j.joep.2011.02.004

Villagómez, Alejandro e Hidalgo, José Antonio (2017), "Financial Literacy and Mathematics: A Study among Young Mexican High School Students", Revista Mexicana de Economía y Finanzas, 12 (2), Ciudad de México, Instituto Mexicano de Ejecutivos de Finanzas (IMEF), pp. 1-22, doi:10.21919/remef.v12i2.88

Villagómez, Alejandro (2016), "Alfabetismo financiero en jóvenes preparatorianos en la Zona Metropolitana del Valle de México", El 
Trimestre Económico, 83 (331), Ciudad de México, Fondo de Cultura Económica, pp. 677-706, doi:10.20430/ete.v83i331.215

Villagómez, Alejandro y González, Alayn (2014), "El efecto del alfabetismo financiero en el ahorro para el retiro en México", documento de trabajo núm. 576, Ciudad de México, CIDE (Centro de Investigación y Docencia Económicas).

Willis, Lauren (2008), "Against Financial Literacy Education”, Iowa Law Review, vol. 94, Rochester, SSRN (Social Science Research Network), pp. 1-197, <https://bit.ly/3a1w4z7>, 4 de diciembre de 2020 .

Recibido: 4 de mayo de 2020. Reenviado: 20 de octubre de 2020. Aceptado: 29 de noviembre de 2020.

Osvaldo García Mata. Maestro en Ciencias en Ingeniería en Sistemas Económicos e Investigación de Operaciones por Stanford University, Estados Unidos. Actualmente es profesor en la Facultad de Comercio y Administración Victoria de la Universidad Autónoma de Tamaulipas, México. Sus líneas de investigación son bienestar financiero y alfabetismo financiero. Entre sus publicaciones más recientes destacan, en coautoría: "La motivación de las mujeres en el desarrollo de emprendimientos rurales y toma de decisiones y la relación con su satisfacción", Recherches en Sciences de Gestion, 6 (135), Lyon, Cairn, pp. 57-77 (2019); "El alfabetismo financiero como determinante del éxito en los micronegocios de las personas que trabajan por su cuenta”, en Elías Alvarado Lagunas y Adriana Guadalupe Chávez Macías (coords.), Los Micronegocios en México: creación, formalización y desafios, Ciudad de México, Pearson Educación de México, pp. 47-72 (2020), y "Actitud, comportamiento y conocimiento financiero en México", Cuadernos de Economía, Bogotá, Universidad Nacional de Colombia (próximamente). 